\title{
Cracking phenomena in lithium-di-silicate glass ceramics
}

\author{
RAJAT BANERJEE \\ Central Glass and Ceramic Research Institute, Kolkata 700 032, India
}

\begin{abstract}
Lithium-di-silicate glass ceramic $\left(\mathrm{Li}_{2} \mathrm{O}, \mathrm{SiO}_{2}\right)$ with uniformly oriented crystals was placed on a Vickers indentation with extrusion axis horizontally parallel to the base axis. The material was rotated through $0^{\circ}-90^{\circ}$ and at each angle a $20 \mathrm{~N}$ load was applied to ascertain the crack path. It was observed that the crack length decreases and the crack deviates from its original path with increasing angle. The deviation of the crack was correlated with the component of the crack driving force and the theoretical strength of the aligned crystals at different angles.
\end{abstract}

Keywords. Lithium-di-silicate; crystals; angle of rotation; crack driving force.

\section{Introduction}

Glass ceramics are polycrystalline materials prepared by controlled crystallization of the crystal phase in glasses. By regulating the temperature in a controlled manner well shaped crystal growth occurs which is basically random in nature. But it is possible to prepare a material having oriented crystal alignment. It is a general perception that mechanical properties of the two materials (random and oriented) differ considerably. Several investigators have studied the effect of oriented crystallization on the mechanical properties of glass ceramics (Mcmillan 1964; Atkinson and Mcmillan 1976). Most of their studies were concerned primarily on the fracture strength of the material. Hasselman and Fulrath (1966) concluded that the difference between the elastic properties of glass and crystal would produce localized mismatch stress concentration which would be deleterious to the fracture strength of the material. But till recently very few studies have been carried out to correlate the crack formation and propagation with such mismatch stress concentration.

The objective of the present study was to correlate the crack developed by Vickers indentation technique during different angles of rotation of the sample with the interfacial stress between the glass and the crystal in $\mathrm{LiO}_{2}-$ $2 \mathrm{SiO}_{2}$ glass ceramic material.

\section{Materials and methods}

Annealed lithium-di-silicate glass ceramic was taken with optical polish on all sides. The direction of the crystals were all uniformly aligned along the extrusion axis of the glass ceramic. A Vickers indentation (Model Shimadzu HMV-2000) was utilized to study the development of the crack pattern by rotation of the samples to different angles. The indenter table consisted of two circular plates with degree 0 to 180 inscribed on it. The lower plate was attached firmly with the indenter table whereas the upper plate was free to rotate. In the beginning the zero degree of the two plates were made to coincide with each other and the sample was placed horizontally with the extrusion axis parallel to the base axis (i.e. parallel to the axis containing zero degree). A load of $20 \mathrm{~N}$ was applied for $10 \mathrm{~s}$ under normal temperature $\left(12 \pm 5^{\circ} \mathrm{C}\right)$ and humidity $(70 \pm 5 \%)$ conditions. Once the indentation was complete the crack length was measured along the path of propagation. This was repeated 5 times to generate 5 data. The upper circular plate was then slowly turned to $10^{\circ}$ while the lower circular plate remained at $0^{\circ}$. The sample now made an angle of $10^{\circ}$ with the base axis. The same procedure was repeated to generate 5 new data. The experiment was continued by rotating the upper plate through the angles $0,10,20, \ldots$ till it reached $100^{\circ}$ when it was discontinued.

\section{Results and discussion}

Being an oriented glass ceramic material having crystal direction along the extrusion axis, two directions viz. perpendicular to the crystal (vertical) and parallel to the crystal (horizontal), were chosen to analyse the data. The sample was placed along the extrusion axis making $0^{\circ}$ with the base. With the increase in the angle of rotation the crack produced by the $20 \mathrm{~N}$ load increased in the vertical direction and simultaneously got reduced in the horizontal direction. Table 1 shows the crack length in both the directions with the angle of rotation. When the data was plotted as a best fit linear curve for the two directions as shown in figure 1 with standard deviation of $95 \%$, it was observed that the crack length in the two directions (horizontal and vertical) became approximately same at an angle of $48^{\circ}$. It was further observed that with increasing angle the crack length increased but got deviated from its original path of propagation in the vertical as 
Table 1. Crack length in vertical and horizontal directions with rotating angles.

\begin{tabular}{lcc}
\hline Angles & $\begin{array}{c}\text { Vertical } \\
\text { length }(\mu \mathrm{m})\end{array}$ & $\begin{array}{c}\text { Horizontal } \\
\text { length }(\mu \mathrm{m})\end{array}$ \\
\hline 0 & $12 \cdot 12$ & $22 \cdot 32$ \\
10 & $12 \cdot 64$ & $21 \cdot 89$ \\
20 & $12 \cdot 85$ & $19 \cdot 43$ \\
30 & $13 \cdot 12$ & $18 \cdot 39$ \\
40 & $14 \cdot 14$ & $16 \cdot 25$ \\
50 & $16 \cdot 44$ & $15 \cdot 89$ \\
60 & $17 \cdot 62$ & 14.99 \\
70 & $18 \cdot 34$ & $14 \cdot 08$ \\
80 & $20 \cdot 15$ & $12 \cdot 74$ \\
90 & $21 \cdot 36$ & $12 \cdot 29$ \\
\hline
\end{tabular}

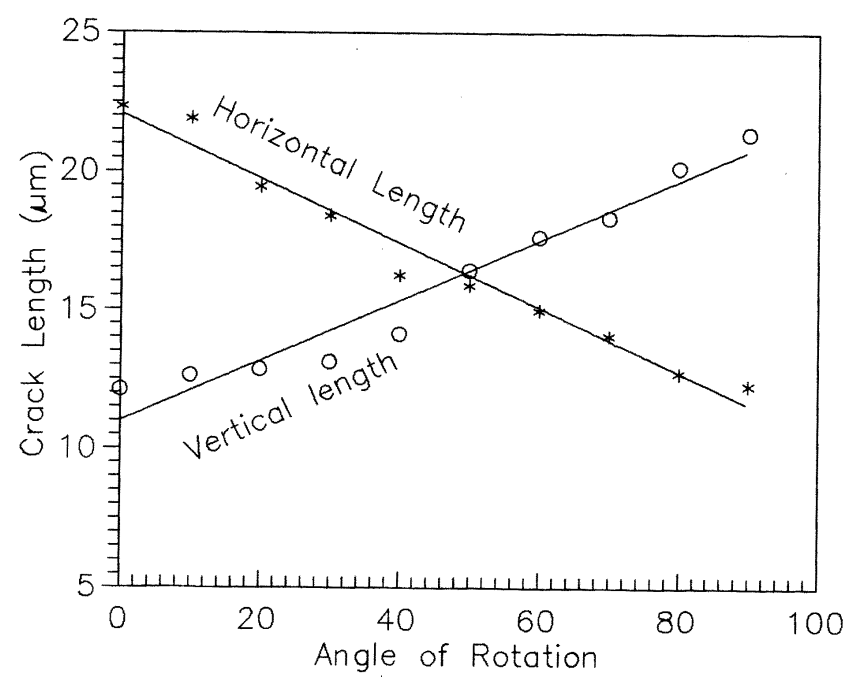

Figure 1. Crack length $(\mu \mathrm{m})$ in both the directions with different angle of rotation. well as in the horizontal directions. Figure 2 clearly shows the deviation of the crack from its original path. At all angles the fracture toughness in two directions viz. vertical and horizontal, was calculated using the formula (Evans and Fuller 1974)

$$
K_{\mathrm{IC}}=0.057 \times H \times \sqrt{ } a \times(E / H)^{2 / 5} \times(a / c)^{3 / 2},
$$

where $a$ is the half diagonal length $(8.4 \mu \mathrm{m}), c$ the crack length from the centre of the indentation in $\mu \mathrm{m}, H$ the Vickers hardness $(6.49 \mathrm{GPa})$ and $E$ the Young's modulus (90 GPa).

With the change in the angle of rotation the crystal orientation also changed which in turn increased/decreased the crack length in the vertical and horizontal directions. Since crack length is inversely proportional to $K_{\text {IC }}$, its values were found to decrease in the vertical direction $v i s-a$-vis in the horizontal direction. The change in the crystal direction with the rotating angle, acted as an hindrance to the propagation of the crack. When the crack propagated parallel to the crystal (horizontal direction at $0^{\circ}$ ) the $K_{\mathrm{IC}}$ was found to be smaller. Actually the measure of the $K_{\text {IC }}$ at this direction gave the measure of the glassy phase in the material. The propagating crack when intersected by the crystals, follow the least resistance path thereby getting deviated from its original path into the glassy phase. The deviation was guided by the component of the crack driving force with the strength of the glassy phase. Table 2 shows the two-component of the crack driving force at different angles of the aligned crystals. As mentioned earlier the quantitative measurement of $K_{\mathrm{IC}}$ in the horizontal direction at an angle of $0^{\circ}$ indicated the strength of the glassy phase, the numerical value of

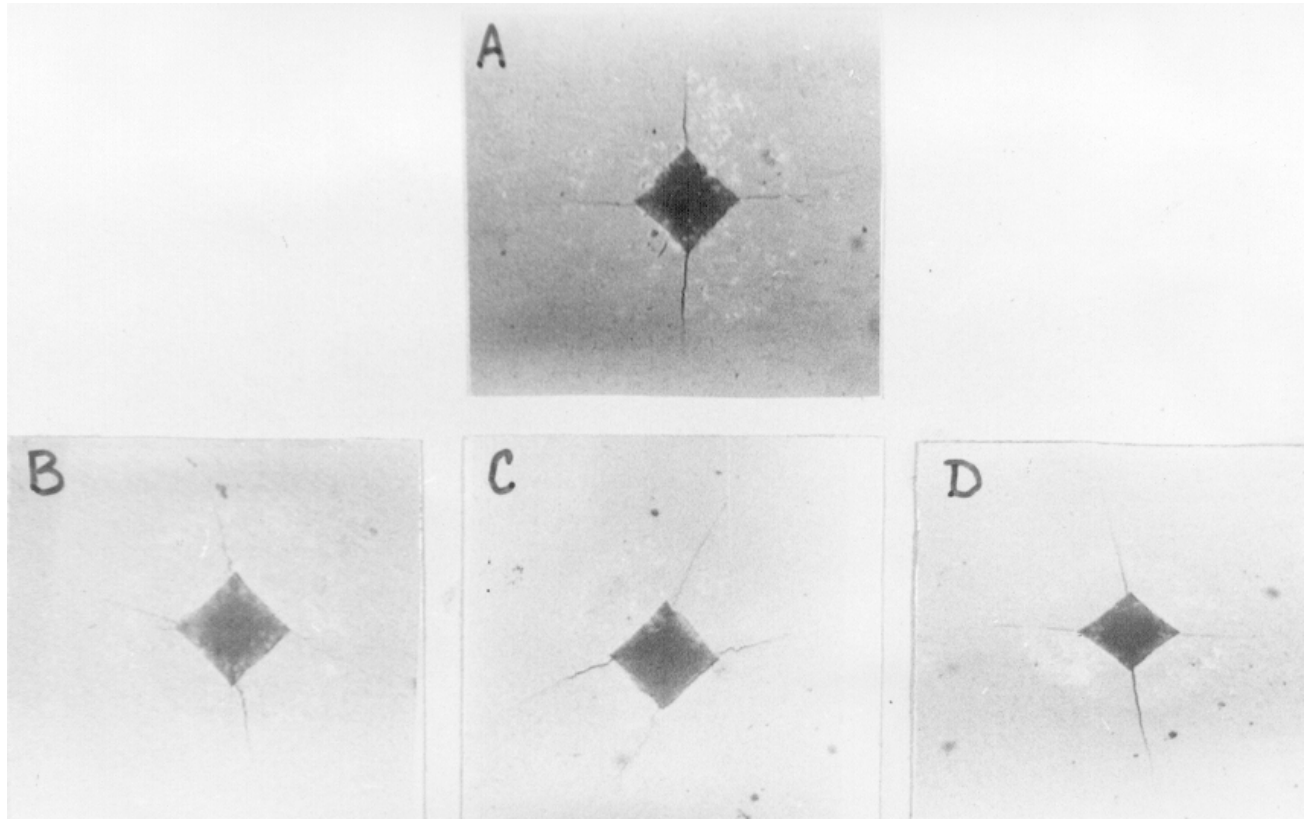

Figure 2. Deviation of the crack path with $20 \mathrm{~N}$ load: A. $0^{\circ}$ angle; B. $30^{\circ}$ angle; C. $50^{\circ}$ angle and D. $80^{\circ}$ angle. 
which was $0.43 \mathrm{MPa} \sqrt{\mathrm{m}}$. Correlating this strength with the components of the crack driving force, it was observed that with increasing angle the crack deviated along the path of the cosine component in the horizontal direction. This phenomena continued approximately to an angle of $45^{\circ}$ after which the numerical value of the sine component became greater than that of the cosine component and the crack deviated along the path of the sine component. The reverse happened in the vertical direction. Figure 3 shows a schematic representation of this deviation, which was somewhat in the form of sinusoidal curve. This was further corroborated from the SEM micrographs at different angles of rotation. The crack length was normalized with respect to $\theta$ where $\theta$ is the angle of rotation. Figure 4 shows the linear dependence of $K_{\mathrm{IC}}$ with $\sqrt{ } 1 /(c / \theta)$ in both the directions. It was apparent that in the vertical direction the data were more scattered than in the horizontal direction due to the misalignment of the crystal in the vertical direction. At $0^{\circ}$ angle the crystal alignment was perpendicular to the propagating crack. Hence there was a wide difference in crack length in the vertical direction than in the horizontal direction. From the slope of the curve one could see that the mean fracture energy needed to initiate the crack was more in the vertical direction $\left(0.21 \mathrm{~J} / \mathrm{m}^{2}\right)$ than in the horizontal direction $\left(0 \cdot 124 \mathrm{~J} / \mathrm{m}^{2}\right)$. Being an anisotropic material the numerical value of the mean fracture energy clearly justifies that the crystal direction plays a dominant role for the crack propagation.

Table 2. Component of crack driving force with rotating angles.

\begin{tabular}{lcccccc}
\hline Angles & $\begin{array}{c}G_{\mathrm{IC}}(\mathrm{V}) \\
(\mathrm{MPa})\end{array}$ & $\begin{array}{c}G_{\mathrm{IC}} V \sin (\theta) \\
(\mathrm{MPa})\end{array}$ & $\begin{array}{c}G_{\mathrm{IC}} V \cos (\theta) \\
(\mathrm{MPa})\end{array}$ & $\begin{array}{c}G_{\mathrm{IC}} H \\
(\mathrm{MPa})\end{array}$ & $\begin{array}{c}G_{\mathrm{IC}} H \sin (\theta) \\
(\mathrm{MPa})\end{array}$ & $\begin{array}{c}G_{\mathrm{IC}} H \cos (\theta) \\
(\mathrm{MPa})\end{array}$ \\
\hline 0 & $8 \cdot 8$ & $0 \cdot 0$ & $8 \cdot 8$ & $4 \cdot 7$ & $0 \cdot 0$ & $4 \cdot 7$ \\
10 & $8 \cdot 5$ & $1 \cdot 4$ & $8 \cdot 3$ & $4 \cdot 8$ & $0 \cdot 8$ & $4 \cdot 7$ \\
20 & $8 \cdot 4$ & $2 \cdot 8$ & $7 \cdot 8$ & $5 \cdot 5$ & $1 \cdot 8$ & $5 \cdot 1$ \\
30 & $8 \cdot 2$ & $4 \cdot 1$ & $7 \cdot 1$ & $5 \cdot 8$ & $2 \cdot 9$ & $5 \cdot 0$ \\
40 & $7 \cdot 6$ & $4 \cdot 8$ & $5 \cdot 8$ & $6 \cdot 7$ & $4 \cdot 3$ & $5 \cdot 1$ \\
50 & $6 \cdot 6$ & $5 \cdot 0$ & $4 \cdot 2$ & $6 \cdot 8$ & $5 \cdot 2$ & $4 \cdot 3$ \\
60 & $6 \cdot 2$ & $5 \cdot 3$ & $3 \cdot 1$ & $7 \cdot 3$ & $6 \cdot 3$ & $3 \cdot 6$ \\
70 & $6 \cdot 0$ & $5 \cdot 6$ & $2 \cdot 0$ & $7 \cdot 7$ & $7 \cdot 2$ & $2 \cdot 6$ \\
80 & $5 \cdot 3$ & $5 \cdot 2$ & $0 \cdot 9$ & $8 \cdot 4$ & $8 \cdot 2$ & $1 \cdot 4$ \\
90 & $5 \cdot 1$ & $5 \cdot 1$ & $0 \cdot 0$ & $8 \cdot 7$ & $8 \cdot 7$ & $0 \cdot 0$ \\
\hline
\end{tabular}
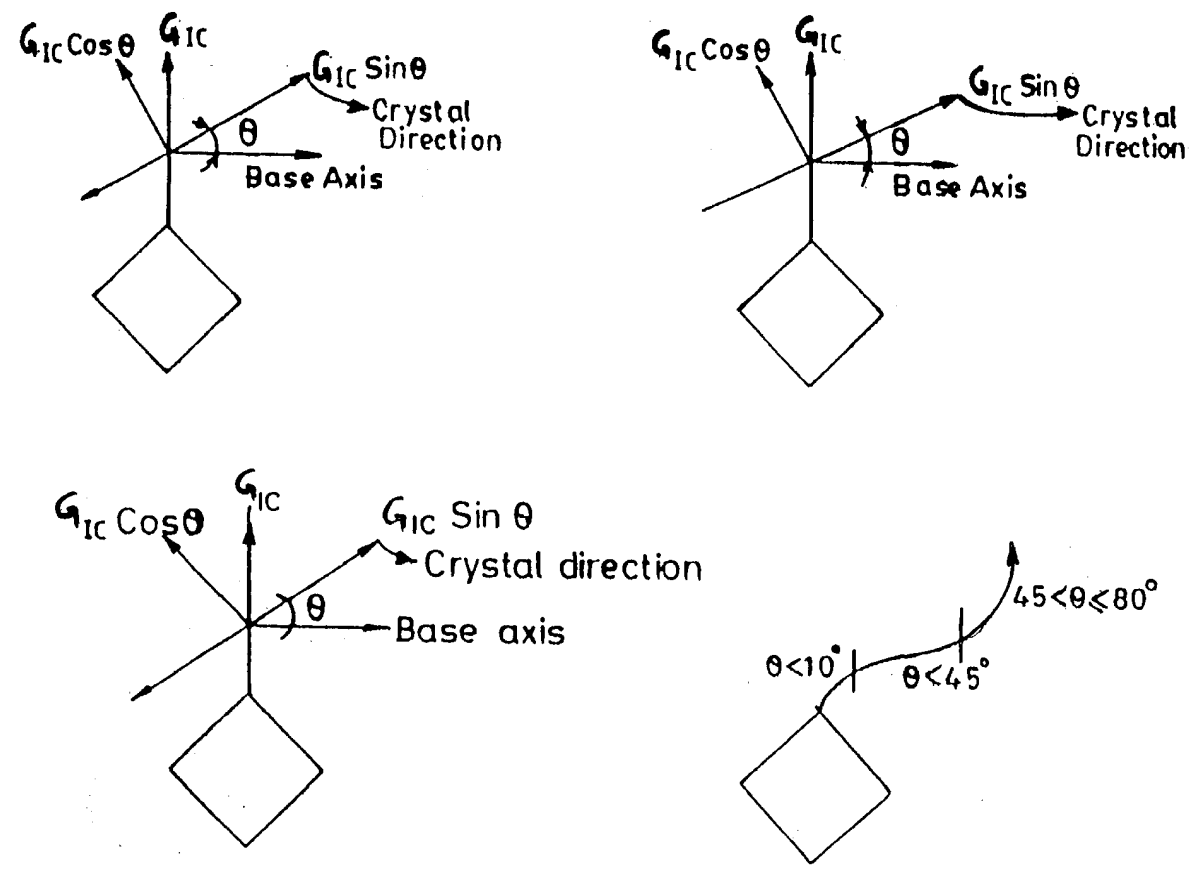

Figure 3. Schematic diagram of deviation of crack length with the crystal direction. 


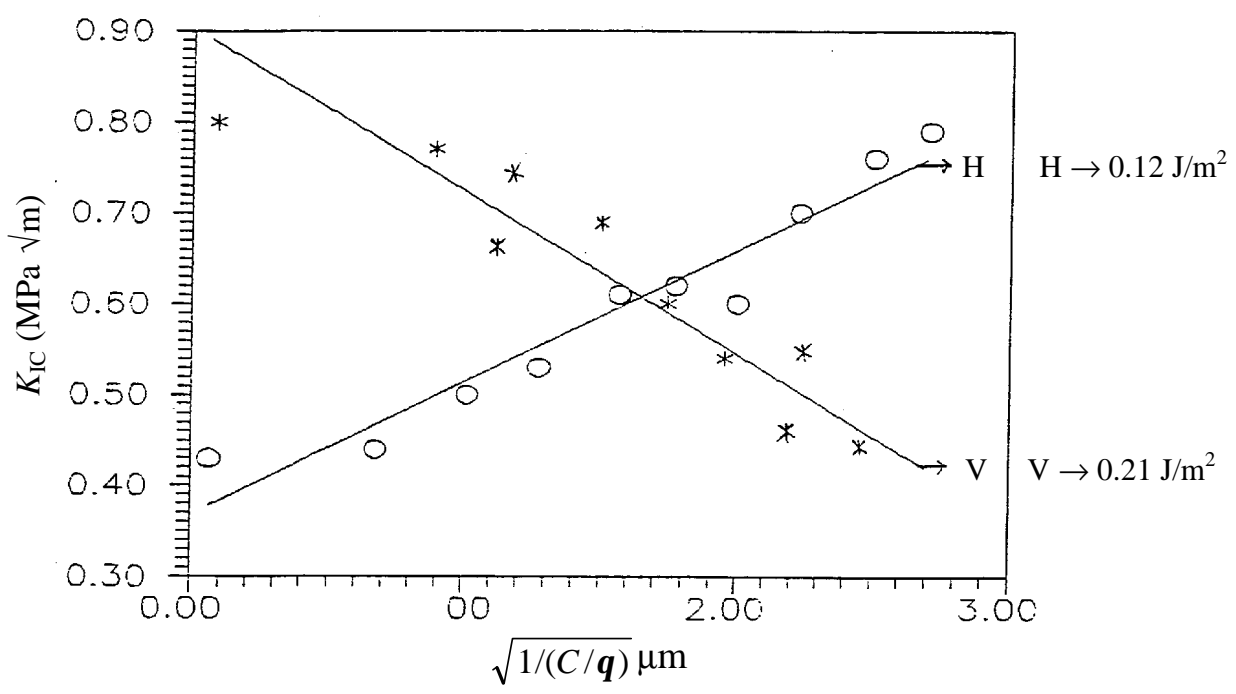

Figure 4. Linear dependence of $K_{\mathrm{IC}}$ with $\sqrt{1 / C}(\theta)$ in both directions.

\section{Conclusion}

In conclusion the crystal direction plays a major role in crack deviation. The deviation being sinusoidal in nature was guided by the component of crack driving force which deviates the crack into the glassy phase since its strength is less compared to that of the crystal.

\section{Acknowledgements}

The author wishes to acknowledge all colleagues of Insti- tut für Materialwissenschaft, FSU, Jena, Germany for academic support and DFG for financial support.

\section{References}

Atkinson D I H and Mcmillan P W 1976 J. Mater. Sci. 11 Pt-1 \& Pt-II 989, 994

Evans A G and Fuller E R 1974 Met. Trans. 527

Freiman S W and Hench L L 1968 J. Am. Ceram. Soc. 51382

Hasselman D P H and Fulrath R M 1966 J. Am. Ceram. Soc. 4968

Mcmillan P W 1964 Glass ceramics (London: Academic Press) p. 132 